\title{
Influencia de la enfermedad renal crónica terminal en la aparición de pérdida de la integridad tisular
}

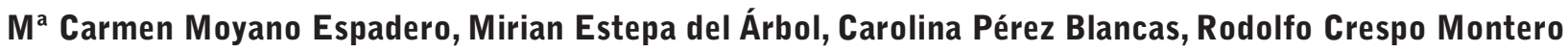 \\ Universidad de Córdoba. Unidad de Gestión Clínica de Nefrología. Hospital Universitario Reina Sofía. Córdoba. \\ España
}

\section{Resumen}

Introducción: La calcifilaxis se caracteriza por calcificación de arteriolas, fibrosis y trombosis que conduce a la aparición de úlceras cutáneas isquémicas y necrosis, y afecta con mayor frecuencia a pacientes con enfermedad renal crónica terminal. El tratamiento de la calcifilaxis es variado, sin existir una modalidad terapéutica eficaz, y presenta mal pronóstico, principalmente por la sepsis secundaria a infección de las lesiones cutáneas.

Objetivo: Conocer la producción científica sobre la calcifilaxis relacionada con la enfermedad renal crónica.

Metodología: Se ha llevado a cabo una revisión bibliográfica con una búsqueda de bibliografía en las principales bases de datos PubMed/Medline, Scielo, Cuiden, ProQuest/Health and Medical Complete, IME y Google Académico.

Resultados: Se seleccionaron 61 artículos para la realización del estudio: 19 artículos de revisión, 13 originales y 29 casos clínicos. Las variables estudiadas han sido las alteraciones en el metabolismo del fósforo, calcio y hormona paratiroidea, varios factores de riesgo, distribución de las lesiones, fallecimiento por sepsis, tratamiento médico y cuidados de enfermería. Los factores más influyentes en la aparición de calcifilaxis son las alteraciones en el metabolismo, obesidad, diabetes, hipertensión, baja albúmina sérica y toma de anticoagulantes. El tratamiento implica múltiples medidas terapéuticas, dirigidas al control de las alteraciones del metabolismo y la cura de las manifestaciones cutáneas.

$$
\begin{gathered}
\text { Correspondencia: } \\
\text { Ma Carmen Moyano Espadero } \\
\text { C/. Poeta Federico García Lorca, n } 34 \\
\text { 14520 Fernán-Núñez. Córdoba } \\
\text { E-mail: mcarmen_me@hotmail.es }
\end{gathered}
$$

Conclusiones: La calcifilaxis es una entidad poco frecuente y con una elevada mortalidad. La patogénesis es desconocida y desde el punto de vista terapéutico, no existe un tratamiento específico, por lo que es fundamental un enfoque multidisciplinar, para su prevención y detección precoz.

\section{PALABRAS CLAVE}

- CALCIFILAXIS

- INSUFICIENCIA RENAL CRÓNICA

- MANIFESTACIONES DÉRMICAS

- DIÁLISIS Y CUIDADOS DE ENFERMERÍA

Influence of chronic kidney disease in the occurrence of loss of tissue integrity

\section{Abstract}

Introduction: Calciphylaxis is characterized by calcification of arterioles, fibrosis and thrombosis which leads to the onset of ischemic skin ulcers and necrosis, and most often affects in patients with end stage renal disease. Treatment of calciphylaxis is varied, without effective therapeutic modality and poor prognosis, mainly due to sepsis secondary to infection of the skin lesions.

Objective: To know the scientific production on calciphylaxis related to chronic kidney disease.

Methodology: A literature review was conducted with a literature search in the following databases: PubMed / Medline, Scielo, Cuiden, ProQuest / Health and Medical Complete, IME and Google Scholar.

Results: Sixty-one articles were selected for the study: 19 review articles, 13 originals and 29 clinical 
cases. The variables studied were: alterations in the metabolism of phosphorus, calcium and parathyroid hormone, several risk factors, distribution of injuries, death due to sepsis, medical treatment and nursing care. The most influential factors in the development of calciphylaxis are alterations in metabolism, obesity, diabetes, hypertension, low serum albumin and taking anticoagulants. Treatment involves multiple therapeutic measures aimed at controlling the metabolic disorders and cure of cutaneous manifestations.

Conclusion: Calciphylaxis is an uncommon event with high mortality. The pathogenesis is unknown and from a therapeutic point of view, there is no specific treatment, so that a multidisciplinary approach is essential for its prevention and early detection.

\section{KEYWORDS}

- CALCIPHYLAXIS

- CHRONIC KIDNEY DISEASE

- SKIN MANIFESTATIONS

- DIALYSIS AND NURSING CARE

\section{Introducción}

\section{Definición}

La calcifilaxis, también conocida como "arteriolopatía calcificante urémica", "síndrome de gangrena urémica" o "paniculitis calcificante" se caracteriza por la calcificación de la capa media de las arteriolas dermoepidérmicas, proliferación de la capa íntima y fibrosis, que predispone a trombosis y a la posterior evolución a úlceras isquémicas y necrosis cutánea ${ }^{1,2,3}$.

Se asocia principalmente a pacientes con enfermedad renal crónica terminal (ERCT) en diálisis, prediálisis y trasplantados 4,5 . Su incidencia se estima en un $1 \%$ y una prevalencia del $1-4 \%$, en pacientes con ERCT y una mortalidad del $60-80 \%{ }^{6}$.

\section{Etiopatogénesis}

La etiopatogénesis se desconoce ${ }^{6}$, sin embargo, se han propuesto algunos posibles mecanismos implicados:

- En pacientes con insuficiencia renal crónica, la disminución de la absorción intestinal del calcio, da lugar a hipocalcemia, lo que determina un aumento en la síntesis de hormona paratiroidea, originando así hiperparatiroidismo secundario. Los niveles elevados de hormona paratiroidea, da lugar a la liberación de calcio y fósforo, normalizando la calcemia y favoreciendo la hiperfosfatemia, que se intensifica debido a la disminución del aclaramiento renal del fósforo en estos pacientes ${ }^{7}$. Los niveles elevados de fósforo inducen la diferenciación osteogénica de las células del músculo liso vascular ${ }^{8}$. Se identifica así un aumento en la expresión de marcadores osteogénicos que participan en la calcificación, entre otros, la osteopontina y proteína ósea morfogénica-4 (BMP-4) y una disminución de inhibidores de la calcificación, como la proteína GLA de la matriz, fetuina-A o alfa 2-Heremans Schmid glycoprotein (AHSG) y pirofosfatos9. El tratamiento con warfarina, se ha considerado un factor implicado en la calcificación vascular, al inhibir la gamma-carboxilación dependiente de vitamina $\mathrm{K}$ de la proteína GLA de la matriz ${ }^{10}$.

- Las especies reactivas de oxígeno (ROS) tienen un papel importante en la calcificación vascular, así como también el eje factor nuclear kappa B (NFkB)-osteoprotegerina/RANK/RANKL; un importante sistema en la homeostasis ósea ${ }^{11}$.

- Las citoquinas proinflamatorias, interleuquina-6 (IL6) y factor de necrosis tumoral alfa (TNF $\alpha$ ), son causa de disfunción endotelial y calcificación vascular ${ }^{12}$.

- La remodelación de la matriz extracelular (MEC) se considera una alteración temprana en el desarrollo de la enfermedad ${ }^{13}$.

- Recientemente se ha informado acerca de la implicación de nanobacterias en el desarrollo de la calcificación vascular ${ }^{14}$.

Para que se manifieste la calcifilaxis, se requiere no solo la calcificación de las arteriolas, sino la disminución del flujo sanguíneo, que conduce a la aparición de úlceras isquémicas. Entre los factores que dan lugar a isquemia se distinguen: El aumento del grosor de la pared vascular, ocupación de la luz del vaso, alteraciones hemodinámicas que provocan disminución del flujo periférico ${ }^{15}$.

Además de la complejidad que plantea la patogénesis de la calcifilaxis, se han identificado factores de riesgo que favorecen el proceso de calcificación vascular, entre ellos, el hiperparatiroidismo, hiperfosfatemia, elevado producto $C a x P$, suplementos de vitamina $D$, bajos niveles de albúmina sérica, deficiencia de proteínas $C$ y $\mathrm{S}$, traumas, como inyecciones subcutáneas (heparina 0 
insulina), tratamiento con warfarina, administración de hierro, corticosteroides, obesidad, sexo femenino, diabetes mellitus, raza blanca, entre otros ${ }^{16,17,18}$.

\section{Manifestaciones clínicas}

Las manifestaciones cutáneas comienzan con áreas de eritema moteado o reticular, similar a livedo reticularis, con nódulos subcutáneos y placas violáceas, purpúricas o equimóticas, dolorosas, que pueden progresar a úlceras necróticas y la formación de escaras. Tienden a ser simétricas, bilaterales y superficiales, y los pulsos arteriales distales permanecen palpables. Estas lesiones pueden tener un patrón de distribución distal (piernas, pies y dedos) o proximal (muslos, nalgas y abdomen), menos frecuente y con peor pronóstico. Se distribuyen especialmente por las extremidades ${ }^{19}$. La calcifilaxis es por tanto, una entidad con muy mal pronóstico, asociado a la mortalidad por sepsis y afectación de órganos ${ }^{20}$.

\section{Tratamiento médico}

Las principales medidas terapéuticas indicadas para el tratamiento de la calcifilaxis son $21,22,23,24,25$ :

- Control de la calcemia e hiperfosfatemia, mediante quelantes de fosfato no basados en calcio, como sevelamer o carbonato de lantano, eliminación o reducción del uso de análogos de la vitamina $\mathrm{D}$, así como una dieta con restricción de fosfato.

- Para el control del hiperparatiroidismo, se puede considerar paratiroidectomía o tratamiento médico con calcimiméticos como cinacalcet. Algunos autores, también han indicado el uso de análogos de vitamina $D$, como el paricalcitol.

- Terapia de reemplazo renal. Aumentar frecuencia y duración de las sesiones de diálisis, con concentraciones bajas de calcio en el líquido de diálisis.

- Evitar medicamentos procoagulantes, así como el uso de warfarina, usando anticoagulantes alternativos, como la heparina de bajo peso molecular.

- Medidas generales como optimizar la nutrición, perfusión tisular, manejo de la anemia, sepsis, control del dolor, evitar traumas, entre otras.

- Tiosulfato de sodio. Se desconoce el mecanismo, pero parece tener un papel importante en la disolución del calcio de los tejidos a partir de la formación de tiosulfato de calcio soluble. Tiene propiedades antioxidantes y contribuye a aliviar el dolor.
- Bifosfonatos. Estos compuestos modifican la cristalización o reabsorción de la hidroxiapatita in vitro y se piensa que pueden tener el mismo efecto in vivo. También actúan inhibiendo la diferenciación de las células precursoras de osteoclastos en células maduras. Tienen efectos antiinflamatorios y contribuyen a disminuir el dolor.

- Cuidado intensivo de las heridas. El desbridamiento es controvertido y en cuanto al uso de apósitos, deben elegirse aquellos que eliminen el exceso de exudado, reduzcan el dolor o eviten trauma al retirarse. Tratar las infecciones con antibióticos. También se puede considerar el injerto de piel o fibroblastos autólogos, el activador de plasminógeno tisular y la terapia con oxígeno hiperbárico.

\section{Objetivos}

Objetivo principal: Conocer la producción científica sobre la calcifilaxis relacionada con la enfermedad renal crónica terminal.

\section{Objetivos específicos:}

- Determinar el conocimiento actual sobre la etiología, patogénesis y tratamiento de la calcifilaxis.

- Determinar el manejo terapéutico adecuado para un cuidado óptimo de las lesiones cutáneas en la calcifilaxis.

\section{Metodología}

Diseño. El diseño de estudio es el de revisión bibliográfica.

Estrategia de búsqueda. La búsqueda de bibliografía se realizó en varias bases de datos con el fin de obtener la mayor producción científica disponible sobre el tema de estudio. Las bases de datos a las que se accedió fueron PubMed/Medline, Scielo, Cuiden, ProQuest/Health and Medical Complete, IME y Google Académico.

Se llevaron a cabo dos búsquedas bibliográficas, siendo el periodo de consulta de la primera búsqueda desde primeros de enero a mediados de febrero y en la segunda de mediados a finales de mayo.

La búsqueda inicial se llevó a cabo en las bases de datos Google Académico, Scielo y Cuiden y posteriormente 
se accedió a la Biblioteca Virtual de la Universidad de Córdoba donde de las diversas bases de datos disponibles, se utilizaron para la búsqueda PubMed/Medline, IME y ProQuest/Health and Medical Complete, mientras que la segunda búsqueda se realizó en la base de datos PubMed/Medline.

El método de búsqueda fue diferente en las distintas bases de datos. Las palabras clave empleadas para la búsqueda fueron: calcifilaxis (calciphylaxis), insuficiencia renal crónica (renal insufficiency chronic), manifestaciones dérmicas (skin manifestations), diálisis (dialysis) y enfermería (nursing).

También se analizó las referencias bibliográficas de algunos artículos con el objetivo de incluir otros estudios relevantes para la presente revisión. La búsqueda de estos artículos se realizó en Google Académico.

\section{Criterios de inclusión:}

- Artículos relacionados con la calcifilaxis en la enfermedad renal crónica.

- Artículos relacionados con manifestaciones en la enfermedad renal crónica que incluyan entre ellas la calcifilaxis.

- Artículos sobre calcificación vascular.

- Artículos con menos de 15 años.

- Artículos en español e inglés.

- Artículos referidos a la edad adulta.

\section{Criterios de exclusión:}

- Artículos sin texto completo disponible.

- Artículos que traten sobre patologías no relacionadas con la enfermedad renal, en las que se desarroIla simultáneamente calcifilaxis.

- Cartas al director.

- Informes breves.

Selección de artículos. Al inicio de la primera búsqueda no se consideró ningún criterio de inclusión/exclusión, para conocer la producción científica existente y obtener mayor conocimiento sobre el tema en cuestión.

Tras la lectura de los títulos, resúmenes y en algunos casos, el artículo completo, se descartaron todos aquellos artículos que no cumplían con los criterios de inclusión/ exclusión (artículos que no tratan del tema de estudio, cartas al director, informes breves, idioma, etc.) y que estuviesen repetidos. En la búsqueda inicial se consideró un período máximo de 10 años en los artículos.
Posteriormente se requirió una segunda búsqueda, en la que se llevó a cabo el mismo proceso que en la búsqueda inicial; seleccionando posteriormente artículos con un mayor período de tiempo, ampliado a 15 años. También se recuperaron algunos de los artículos descartados en la búsqueda inicial, teniendo en cuenta los criterios de inclusión/exclusión.

En la primera búsqueda se identificaron, con el total de combinaciones de términos Mesh, 905 artículos en las bases de datos, de los cuales se seleccionaron 47 artículos tras aplicar los criterios de inclusión/exclusión y descartar artículos repetidos. En la segunda búsqueda se identificaron un total de 193 artículos en la base de datos PubMed, de los cuales se seleccionaron 6 artículos, con las mismas consideraciones que la búsqueda anterior y se recuperó un total de 10 artículos de la búsqueda inicial. Finalmente se seleccionaron, como resultado de ambas búsquedas, un total de 63 artículos.

Durante el proceso de selección, se identificaron artículos sobre casos clínicos, revisiones y artículos originales.

Análisis de artículos. De los artículos finalmente seleccionados, se recopiló información relacionada con la calcifilaxis (definición, patogénesis, manifestaciones clínicas y tratamiento) y datos de los casos clínicos y originales para el posterior análisis de variables de estudio.

Para el análisis de las variables relacionadas con el metabolismo (fósforo, calcio, producto CaxP, hormona paratiroidea) y albúmina, sobre las cuales no se tenía información acerca de valores bioquímicos de referencia o no se hacía mención en el artículo a la interpretación de tales valores, se utilizaron los parámetros recomendados para el estadio $\mathrm{V}$ de la enfermedad renal crónica, de la guía "Recomendaciones de la Sociedad Española de Nefrología para el manejo de las alteraciones del metabolismo óseo-mineral en los pacientes con enfermedad renal crónica (S.E.N.MM)" y las guías de la Fundación Nacional del Riñón: "KDOQI Clinical Practice Guidelines for Bone Metabolism and Disease in Chronic Kidney Disease" y "Clinical Practice Guidelines for Nutrition in Chronic Renal Failure":

- Calcio: 8.4-9.5 mg/dL (2.1-2.37 mmol/L)

- Fósforo: $3.5-5.5 \mathrm{mg} / \mathrm{dL}(1.13-1.78 \mathrm{mmol} / \mathrm{L})$

- Producto CaxP $<55 \mathrm{mg}^{2} / \mathrm{dL}^{2}$

- Hormona paratiroidea: 150-300 pg/mL (16.5-33 $\mathrm{pmol} / \mathrm{L}$ )

- Albúmina: $\geq 4 \mathrm{~g} / \mathrm{dL}$ 


\section{Resultados}

Los artículos encontrados en la búsqueda inicial con la combinación de términos Mesh, se indican en la tabla I. Los artículos seleccionados son el resultado del descarte de artículos que no cumplían con los criterios de inclusión/exclusión, considerando inicialmente como período de tiempo un máximo de 10 años, y artículos repetidos.

Tabla I. Artículos seleccionados para el estudio en la $1^{\text {a }}$ búsqueda.

\begin{tabular}{|l|c|c|c|c|c|c|c|}
\hline Bases de datos & PubMed & Health and Medical Complete & IME & Cuiden & Scielo & Google Académico & Total artículos \\
\hline Artículos encontrados & 343 & 44 & 19 & 13 & 28 & 458 & 905 \\
\hline Artículos seleccionados & 26 & 4 & 3 & 2 & 4 & 8 & 47 \\
\hline
\end{tabular}

Tras la búsqueda inicial, se llevó a cabo una segunda búsqueda, donde se encontraron los resultados expuestos en la tabla II.

Los artículos descartados en la búsqueda inicial y posteriormente recuperados ampliando el período de tiempo a 15 años, se exponen en la tabla III.
Tabla II. Artículos seleccionados para el estudio en la $2^{\mathrm{a}}$ búsqueda.

\begin{tabular}{|l|c|}
\hline Base de datos & PubMed \\
\hline Artículos encontrados & 193 \\
\hline Artículos seleccionados & 6 \\
\hline
\end{tabular}

Tabla III. Artículos seleccionados para el estudio recuperados de la búsqueda inicial.

\begin{tabular}{|c|c|c|c|c|c|c|c|}
\hline Bases de datos & PubMed & Health and Medical Complete & IME & Cuiden & Scielo & Google Académico & Total artículos \\
\hline $\begin{array}{l}\text { Artículos } \\
\text { Encontrados }\end{array}$ & 343 & 44 & 19 & 13 & 28 & 458 & 905 \\
\hline $\begin{array}{l}\text { Artículos } \\
\text { preseleccionados }\end{array}$ & 26 & 4 & 3 & 2 & 4 & 8 & 47 \\
\hline $\begin{array}{l}\text { Artículos } \\
\text { seleccionados }\end{array}$ & 28 & 7 & 6 & 2 & 4 & 10 & 57 \\
\hline
\end{tabular}

El total de artículos seleccionados entre la búsqueda inicial y la segunda búsqueda es de 63 artículos y se muestra en la tabla IV.

Tabla IV. Artículos seleccionados para el estudio de la $1^{\circ}$ y $2^{\circ}$ búsqueda.

\begin{tabular}{|l|c|}
\hline Búsquedas & Total \\
\hline $\mathbf{1}^{\text {a }}$ Búsqueda & 57 \\
\hline $2^{\text {a }}$ Búsqueda & 6 \\
\hline Total & 63 \\
\hline
\end{tabular}

Finalmente los artículos seleccionados para la realización de la revisión se muestran en las siguientes tablas, clasificadas según el tipo de artículo.
Tabla V. Revisiones bibliográficas.
Autor, año, país

Diseño del estudio

\begin{tabular}{|c|c|}
\hline Autor, año, país & Diseño del estudio \\
\hline Cordova KB et al. 2009. EEUU. ${ }^{4}$ & Revisión \\
\hline Galperin TA et al. 2014. EEUU. ${ }^{6}$ & Revisión \\
\hline Karwowski W et al. 2012. Polonia. ${ }^{8}$ & Revisión \\
\hline Brewster UC. 2008. EEUU.? & Revisión \\
\hline Virmani R et al. 2014. EEUU. ${ }^{10}$ & Revisión \\
\hline Sowers KM et al. 2010. EEUU. ${ }^{11}$ & Revisión \\
\hline Brandenburg VM et al. 2012. Alemania. ${ }^{13}$ & Revisión \\
\hline Hayden MR et al. 2005. EEUU. ${ }^{14}$ & Revisión \\
\hline Fernández E. 2008. España. ${ }^{15}$ & Revisión \\
\hline Mathur RV et al. 2001. Reino Unido. ${ }^{16}$ & Revisión \\
\hline Llach F. 2001. EEUU. ${ }^{17}$ & Revisión \\
\hline Panuncialman J et al. 2010. EEUU. ${ }^{18}$ & Revisión \\
\hline
\end{tabular}


Tabla V. Revisiones bibliográficas. (continuación)

\begin{tabular}{|c|c|}
\hline Autor, año, país & Diseño del estudio \\
\hline Pastor-Nieto MA. 2001. España. ${ }^{19}$ & Revisión \\
\hline Brewster UC. 2006. EEUU. ${ }^{20}$ & Revisión \\
\hline Ross EA. 2011. EEUU. ${ }^{21}$ & Revisión \\
\hline Ong $S$ et al. 2012. Reino Unido. ${ }^{22}$ & Revisión \\
\hline Rogers NM et al. 2007. Australia. ${ }^{23}$ & Revisión \\
\hline Polaina-Rusillo M y cols. 2009. España. ${ }^{24}$ & Revisión \\
\hline Bhambri A et al. 2008. EEUU. ${ }^{25}$ & Revisión \\
\hline
\end{tabular}

Tabla VI. Casos clínicos.

\begin{tabular}{|c|c|c|}
\hline Autor, año, país & Muestra & Diseño del estudio \\
\hline $\begin{array}{l}\text { Gómez de la Fuente E y cols. } \\
\text { 2004. España. }{ }^{2}\end{array}$ & 4 & Casos clínicos \\
\hline $\begin{array}{l}\text { Rodríguez-Villarreal I y cols. } \\
\text { 2010. España. }\end{array}$ & 1 & Caso clínico \\
\hline $\begin{array}{l}\text { Prados-Soler } M^{a} C \text { y cols. } \\
\text { 2010. España. }{ }^{5}\end{array}$ & 1 & Caso clínico \\
\hline $\begin{array}{l}\text { García-Villasur P y cols. } \\
\text { 2007. España.7 }\end{array}$ & 1 & Caso clínico \\
\hline $\begin{array}{l}\text { Zapata-González F y cols. } \\
\text { 2013. Colombia. }{ }^{12}\end{array}$ & 1 & Caso clínico \\
\hline $\begin{array}{l}\text { Picazo M y cols. } \\
\text { 2005. España. }\end{array}$ & 1 & Caso clínico \\
\hline $\begin{array}{l}\text { Capote-Moreno A y cols. } \\
\text { 2002. España. }{ }^{28}\end{array}$ & 5 & Casos clínicos \\
\hline $\begin{array}{l}\text { Munar MA y cols. } \\
\text { 2001. España. }{ }^{29}\end{array}$ & 1 & Caso clínico \\
\hline $\begin{array}{l}\text { Marrón B y cols. } \\
\text { 2001. España..0 }\end{array}$ & 4 & Casos clínicos \\
\hline $\begin{array}{l}\text { Pérez JE y cols. } \\
\text { 2011. Colombia. }\end{array}$ & 1 & Caso clínico \\
\hline $\begin{array}{l}\text { Herrera L y cols. } \\
\text { 2013. Colombia. }\end{array}$ & 1 & Caso clínico \\
\hline $\begin{array}{l}\text { Batún-Garrido JAJ y cols. } \\
\text { 2014. México. }{ }^{33}\end{array}$ & 1 & Caso clínico \\
\hline $\begin{array}{l}\text { Morales-Jiménez EM y cols. } \\
\text { 2013. Cuba. }\end{array}$ & 1 & Caso clínico \\
\hline $\begin{array}{l}\text { Rojas-Estrada JJ y cols. } \\
\text { 2011. Cuba. }{ }^{35}\end{array}$ & 1 & Caso clínico \\
\hline $\begin{array}{l}\text { Piña-Simón D y cols. } \\
\text { 2010. España. }{ }^{36}\end{array}$ & 1 & Caso clínico \\
\hline $\begin{array}{l}\text { Oikawa S et al. } \\
\text { 2004. Japón. }{ }^{37}\end{array}$ & 1 & Caso clínico \\
\hline $\begin{array}{l}\text { Maroulis JC et al. } \\
\text { 2006. Grecia.. }\end{array}$ & 1 & Caso clínico \\
\hline
\end{tabular}

Tabla VI. Casos clínicos. (continuación)

\begin{tabular}{lcc}
\hline Autor, año, país & Muestra & Diseño del estudio \\
$\begin{array}{l}\text { Kakagia D et al. } \\
\text { 2011. Grecia. }{ }^{39}\end{array}$ & 1 & Caso clínico \\
\hline $\begin{array}{l}\text { Jacobs-Kosmin D et al. } \\
\text { 2007. EEUU. }{ }^{40}\end{array}$ & 1 & Caso clínico \\
\hline $\begin{array}{l}\text { Roncada EV et al. } \\
\text { 2012. Brasil.41 }\end{array}$ & 1 & Caso clínico \\
\hline $\begin{array}{l}\text { Marques SA et al. } \\
\text { 2013. Brasil. }{ }^{42}\end{array}$ & 1 & Caso clínico \\
\hline $\begin{array}{l}\text { Hayashi M. } \\
\text { 2013. Japón. }\end{array}{ }^{43}$ & 1 & Caso clínico \\
\hline $\begin{array}{l}\text { Mohammed IA et al. } \\
\text { 2008. Reino Unido. }{ }^{44}\end{array}$ & 1 & Caso clínico \\
\hline $\begin{array}{l}\text { Velasco N et al. } \\
\text { 2006. Reino Unido. }{ }^{45}\end{array}$ & 1 & Caso clínico \\
\hline $\begin{array}{l}\text { Bliss DE. } \\
\text { 2002. EEUU. }{ }^{46}\end{array}$ & 1 & Caso clínico \\
\hline $\begin{array}{l}\text { Headley CM et al. } \\
\text { 2002. EEUU. }{ }^{47}\end{array}$ & 1 & Caso clínico \\
\hline $\begin{array}{l}\text { Garini G et al. } \\
\text { 2006. Italia. }{ }^{48}\end{array}$ & 1 & Caso clínico \\
\hline $\begin{array}{l}\text { Carter T et al. } \\
\text { 2013. EEUU. }{ }^{49}\end{array}$ & 1 & Caso clínico \\
\hline $\begin{array}{l}\text { Sprague SM. } \\
\text { 2014. EEUU. }{ }^{50}\end{array}$ & 1 & \\
\hline & & \\
\hline
\end{tabular}

Tabla VII. Artículos originales.

\begin{tabular}{|c|c|c|}
\hline Autor, año, país & Muestra & Diseño del estudio \\
\hline $\begin{array}{l}\text { Verdalles-Guzmán U y cols. } \\
\text { 2008. España. }{ }^{1}\end{array}$ & 8 & Estudio retrospectivo \\
\hline $\begin{array}{l}\text { Esteve V y cols. } \\
\text { 2007. España. }{ }^{26}\end{array}$ & 8 & Análisis descriptivo \\
\hline $\begin{array}{l}\text { Hayashi M et al. } \\
\text { 2012. Japón. }{ }^{.1}\end{array}$ & 84 & $\begin{array}{l}\text { Estudio de casos y } \\
\text { controles retrospectivo }\end{array}$ \\
\hline $\begin{array}{l}\text { González-Horna A y cols. } \\
\text { 2005. España. }{ }^{52}\end{array}$ & NC & $\begin{array}{l}\text { Estudio } \\
\text { observacional retrospectivo }\end{array}$ \\
\hline $\begin{array}{l}\text { Torregrosa JV y cols. } \\
\text { 2012. España. }{ }^{33}\end{array}$ & 8 & Estudio prospectivo \\
\hline $\begin{array}{l}\text { Mazhar AR et al. } \\
\text { 2001. EEUU. }{ }^{44}\end{array}$ & 73 & $\begin{array}{l}\text { Estudio de casos y } \\
\text { controles retrospectivo }\end{array}$ \\
\hline $\begin{array}{l}\text { Farah M et al. } \\
\text { 2011. Canadá. }{ }^{55}\end{array}$ & 12 & Estudio de cohortes \\
\hline $\begin{array}{l}\text { Fine A et al. } \\
\text { 2008. Canadá. }{ }^{56}\end{array}$ & 59 & Estudio prospectivo \\
\hline $\begin{array}{l}\text { Hammawa-Malabu U et al. } \\
\text { 2012. Australia. }{ }^{57}\end{array}$ & 6 & Estudio retrospectivo \\
\hline
\end{tabular}


Tabla VII. Artículos originales. (continuación)

\begin{tabular}{lcc}
\hline Autor, año, país & Muestra & $\begin{array}{c}\text { Diseño del estudio } \\
\text { New N et al. }\end{array}$ \\
2011. Australia. ${ }^{58}$ & 5 & de cohortes retrospectivo \\
\hline $\begin{array}{l}\text { Nigwekar SU et al. } \\
\text { 2013. EEUU. }{ }^{59}\end{array}$ & 172 & Estudio de cohortes \\
\hline Zitt E et al. & & $\begin{array}{c}\text { Estudio de cohortes } \\
\text { 2013. Austria. }{ }^{60}\end{array}$ \\
\hline $\begin{array}{l}\text { Podymow T et al. } \\
\text { 2001. Canadá. }\end{array}$ & 27 & retrospectivo y multicéntrico \\
\hline
\end{tabular}

\section{Descripción de los resultados}

\section{Fósforo}

Una gran proporción de casos presentaban niveles elevados de fósforo al diagnóstico $3,5,7,27,28,30,31,33,34,35,38,39,40,4$ $1,42,44,45,46,47,48,49$, así como en los originales $26,51,53,54,58,59,61$. Sin embargo, también se ha identificado calcifilaxis en algunos casos clínicos sin alteraciones de los niveles de fósforo $12,29,30,36,37,50,60,61$ y en algunas series de ca$\operatorname{sos}^{1,26,53,55,57}$. Se debe considerar que muchos de ellos tomaban tratamiento para la reducción de niveles de fósforo y/o habían sido sometidos a paratiroidectomía, previamente al diagnóstico.

\section{Calcio}

La mayoría de los casos clínicos presentaban un nivel de calcio normalizado $3,5,12,27,28,29,31,33,34,36,37,38,41,48,50$, igual que algunas series de $\operatorname{casos}^{1,26,53,54,55,57,58,59,60,61}$, así como también se han descrito casos con calcio bajo $28,35,39,40$ y series $^{61}$. Una minoría de $\operatorname{casos}^{7,28,30,42,44,45,46,47}$, así como en originales ${ }^{26,51,53,56,58}$, presentó un nivel de calcio elevado en el momento del diagnóstico.

Una proporción considerable de los casos revisados se encontraba en tratamiento con quelantes de fósforo basados en calcio, suplementos de calcio, análogos de la vitamina $D$ y/o sales de calcio previo al diagnóstico de calcifilaxis, tanto en los casos clínicos $3,5,7,12,28,29,30,33,35,38,39,40,41,44,45$, como en las se-

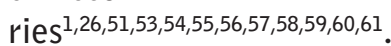

\section{Producto CaxP}

En la mayoría de los casos revisados los pacientes presentaron un producto CaxP eleva$\mathrm{do}^{2,27,28,30,33,34,38,40,42,45,47,48,49}$, así como en algunos de los artículos originales $26,53,54$, sin embargo no todos presentan estos valores, encontrándose un producto CaxP normalizado en algunos $\operatorname{casos}^{29,30,35,36,37,41}$ y series de $\operatorname{casos}^{1,26,53,57,61}$, aunque teniendo en cuenta las mismas consideraciones anteriormente mencionadas.

\section{Hormona paratiroidea}

La mayoría de casos revisados han presentado elevados niveles de hormona paratiroidea $2,7,12,27,28,29,30,31,32$, $33,38,39,40,41,42,44,45,47,48,49,50$, así como los estudios originales ${ }^{1,26,51,52,53,54,57,58,59,60,61}$, sin embargo, también se han encontrado niveles de parathormona sin alteración, tanto en los casos $3,5,35,36,37$ como en las series publicadas $1,26,53,55,58,61$, considerando igualmente que muchos de estos casos estaban recibiendo previamente tratamiento o habían sido intervenidos de paratiroidectomía.

\section{Género}

La mayoría de los casos identificados con calcifilaxis eran del sexo femenino $2,3,5,12,27,28,29,30,31,32,36,39,41,44,46,48,49$, así como en los estudios de $\operatorname{casos}^{1,26,51,53,54,55,56,57,58,59,60,61,}$ aunque también se presenta en una proporción considerable en el sexo masculino, tanto en los ca-

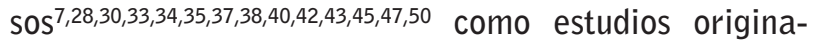
les $^{26,51,53,54,55,56,57,58,59,60,61}$.

\section{Edad}

En los casos estudiados las edades estaban comprendidas principalmente entre los 50-70 años $2,3,5,12$ $, 27,28,29,30,32,33,34,35,39,41,43,46,47,50$, así como en los originales $1,26,51,52,53,54,55,57,58,59,60,61$. En una minoría de casos la calcifilaxis se observó en pacientes con 50 años o menos $^{28,31,37,38,40,45,48,49}$ y con 70 años o más $2,7,28,44,36,42$.

\section{Obesidad}

En los casos clínicos revisados, hay un número importante de casos de calcifilaxis que se asocian con obesidad $2,5,12,27,30,37,41,44,46$, así como en las series de casos $1,55,60,61$.

\section{Diabetes}

En los artículos revisados, se han encontrado bastantes casos de calcifialxis donde el paciente presentaba diabetes mellitus $2,5,12,27,32,33,41,43,44,46,50$, al igual que en los estudios originales $1,51,52,54,55,56,57,58,59,60,61$; a señalar que la mayoría de ellos presentaba diabetes tipo II.

\section{Hipertensión arterial}

De los casos revisados, una gran mayoría presentaba

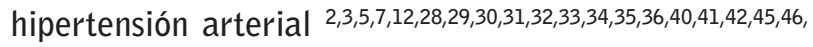
47,49,50. También se han encontrado en los artículos con series más amplias $1,26,54,57,58,60$. 


\section{Raza}

Se observa una mayor frecuencia de la raza blanca entre los casos revisados, de los cuales se tiene constancia de la misma $34,38,39,40,41,43,44,50$, así como en los pacientes de estudios originales $1,54,59,61$, frente a una minoría de pacientes identificados de raza negra entre los casos clínicos $^{46,49}$ y en estudios originales ${ }^{54,59}$.

\section{Albumina sérica \\ Bajos niveles de albúmina sérica también se ha identificado en los pacientes de los casos revisa- $\operatorname{dos}^{12,27,36,37,38,40,41,44,50}$ y originales ${ }^{1,51,53,54,59,60,61}$.}

\section{Pérdida de peso}

En algunos casos se identificó una pérdida de peso previa al desarrollo de calcifilaxis, aunque esto se observó en una minoría $30,37,38,44$.

\section{Anticoagulantes}

Se ha observado en la mayoría de pacientes el uso de algún anticoagulante, normalmente anticoagulantes cumarínicos como la warfarina, previo a la presentación de calcifilaxis $3,5,29,30,36,39,43$, igualmente entre los pacientes de las series más amplias $1,26,51,52,53,54,55,59,60,61$.

\section{Esteroides}

De los casos estudiados, algunos se encontraban en tratamiento con esteroides previo a la aparición de la calcifilaxis 3,30,37, así como en pacientes de estudios originales ${ }^{1,26,53,54,55}$.

\section{Traumatismos}

Del total de casos revisados, sólo en dos pacientes se ha identificado una posible asociación con la aparición de lesiones en la zona donde previamente habían sufrido un traumatismo previo ${ }^{37,40}$.

\section{Modalidad de diálisis}

En la mayoría de los casos revisados, los pacientes se encontraban en hemodiálisis $2,3,5,12,27,28,29,30,32,34,35,36,37,39$,

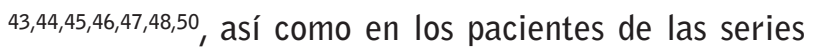
amplias $1,26,53,54,55,56,57,59,60,61$; mientras que los pacientes que estaban en diálisis peritoneal se encuentran en menor proporción tanto en los $\operatorname{casos}^{2,30,31,33,38,42,49}$, como en los originales $55,56,58,60,61$.

\section{Distribución de las lesiones}

En la mayoría de los casos, los pacientes presentaban principalmente lesiones de distribución distal 2,3,5,7,28,3 $0,33,34,36,41,42,45,47,49$, así como en las series más amplias
1,26,53,54,56,57,58,59,60,61 o ambas simultáneamente, distales y

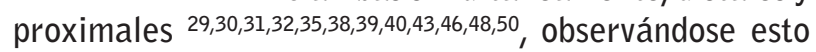
también en los artículos originales 53,54,57,58,59,61. La distribución proximal estaba presente en menor proporción tanto en los $\operatorname{casos}^{12,27,37,44}$, como en las series de $\operatorname{casos}^{1,26,53,54,56,59,60,61}$.

\section{Fallecimiento por sepsis de origen cutáneo}

Se han comunicado bastantes $\operatorname{casos}^{2,5,28,31,33,35,40,42,46}$ de fallecimiento por sepsis de origen cutáneo, como complicación sistémica de la infección de las lesiones que origina la calcifilaxis. De igual forma se han comunicado en estudios con series más amplias ${ }^{1,26,54,57,60,61}$.

\section{Tratamiento médico}

\section{Control del fósforo y calcio}

Las medidas adoptadas en la mayoría de los casos han consistido en el control de las alteraciones del metabolismo. El control de los niveles elevados de fósforo y calcio (dieta, tratamiento con quelantes de fósforo, suspensión de tratamientos como análogos de vitamina D, quelantes cálcicos, etc.) se ha llevado a cabo en la mayoría de los casos 2,3,12,27, 29,31,33,34,35,38,39,40,41,42,44,45,48,4 9,50, y estudios de casos $1,54,56,57,58,59,60$, así como se han realizado ajustes en las sesiones de diálisis en los ca-

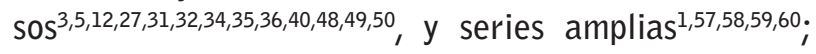
en muchos de ellos, también ajustes con bajo calcio en el líquido dializado tanto en los casos 2,3,5,36,38,39,40,45,49 como estudios de $\operatorname{casos}^{54,57,59,60}$.

\section{Control hormona paratiroidea}

Para el control de niveles elevados de hormona paratiroidea, la paratiroidectomía ha sido una alternativa empleada entre los casos revisados $2,28,29,30,36,37,38,42,46,48$ y

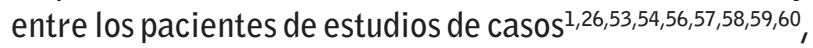
tanto previamente como tras el diagnóstico de calcifilaxis.

\section{Tiosulfato de sodio y bifosfonatos}

Otros tratamientos médicos empleados con frecuencia han sido el tiosulfato de sodio, en los $\operatorname{casos}^{3,5,12,36,43,49,50}$ y series más amplias $57,58,59,60$ y bifosfonatos, en casos revisados $3,5,33,48$ y estudios de $\operatorname{casos}^{1,53,58}$.

\section{Antibióticos y analgésicos}

Otras medidas terapéuticas aplicadas han sido los antibióticos sistémicos y/o tópicos, tanto los ca-

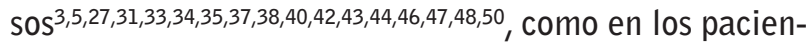
tes de los artículos originales $26,52,54,57,60,61$. 
También se han aplicado analgésicos, en su distintas formas de presentación (tópicos, en parches, vía sistémica, etc.) en los casos $3,5,27,31,32,34,36,37,38,39,40,41,44,46,47,48,49$ y en los pacientes de estudios de casos $52,54,60$.

\section{Terapia de oxígeno hiperbárico y desbridamiento de lesiones}

Se ha aplicado en algunos casos la terapia con oxígeno hiperbárico 3,37 , y en algunas series ${ }^{57,58,60,61}$, así como el desbridamiento de las lesiones, incluyendo desbridamientos quirúrgicos, químicos, autolíticos y/o biológicos (larvas estériles) también se ha realizado en $\operatorname{casos}^{2,3,}$ $7,12,27,30,31,32,34,37,39,40,41,42,43,46,48$ y pacientes de grupos amplios $^{1,26,52,54,57,60,61}$.

\section{Cuidados de enfermería}

Para el manejo local de las lesiones no existe un protocolo estándar, aunque se observa entre los casos revi-

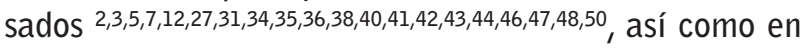
los estudios más amplios $26,52,54,57,59$, de los que se tiene constancia de la curación de las lesiones, unas pautas de actuación comunes, que pueden resumirse de forma general en:

- Medir el tamaño de las úlceras, medidas higiénicas, posturales y curas locales.

- Valorar signos de infección y ante evidencia de infección local o como cobertura profiláctica, usar pomadas antibióticas. En caso de infección, administrar antibióticos por vía oral o intravenosa, previamente prescritos.

- Administrar analgesia, y valorar si realizar la cura intra o posthemodiálisis según tolerancia, estableciendo la periodicidad de las mismas.

- Limpieza de la herida, uso de antisépticos tópicos, colocación de apósitos hidrocoloides.

\section{Discusión}

El objetivo de la presente revisión ha sido conocer la literatura científica sobre la calcifilaxis, que es una entidad poco frecuente de evolución incierta y que puede ocasionar la muerte de quien la padece, aunque el objeto de nuestro interés inicial era saber algo más sobre un tipo de úlceras de difícil manejo, que se diferencian de otras lesiones ulcerosas.

Se atribuye un papel fundamental a las alteraciones del metabolismo del calcio, fósforo, producto CaxP y hormona paratiroidea, en el desarrollo de calcifilaxis, ya que se observa que los niveles de estos parámetros suelen estar elevados en la mayoría de los pacientes que desarrollan esta entidad, en el contexto de la enfermedad renal crónica avanza$\mathrm{da}^{62}$. No obstante, también se han identificado, en informes de casos y estudios, pacientes en los que estos niveles se encontraban dentro del rango normal; lo que genera cierta incertidumbre acerca de la única implicación de estas alteraciones metabóli$\operatorname{cas}^{27-30,35-38,57-61}$.

Con respecto a los factores de riesgo identificados (género, obesidad, diabetes, hipertensión, pérdida de peso, esteroides, etc.), se observa que muchos de ellos están presentes en los casos y originales estudiados, en mayor o menor medida, aunque aún no se ha establecido su verdadera implicación en la calcifilaxis, sino que se basan en especulaciones sobre su posible contribución al desarrollo de la misma ${ }^{50,58}$.

En cuanto a la modalidad de diálisis, se indica la diálisis peritoneal como factor de riesgo para el desarrollo de calcifilaxis ${ }^{43,49,50,51,55,56,58}$, sin embargo, la mayoría de los casos y pacientes de originales revisados, se encontraban en hemodiálisis frente a una minoría en diálisis peritoneal.

En relación al patrón de distribución de las lesiones cutáneas, en una gran proporción de casos y en originales, están presentes a nivel distal e incluso la presencia de ambos ${ }^{31-35,41-43,57,58}$.

Las medidas terapéuticas observadas para el tratamiento de la calcifilaxis en los distintos informes de casos y estudios son múltiples ${ }^{17,21-23,43,50,58,62}$, y ninguna de ellas consistentes en la actualidad, ya que en algunos de estos casos las terapias aplicadas son eficaces y por el contrario, en otros no se logran tales resultados. Lo habitual es combinar varias medidas terapéuticas, para la resolución tanto de la calcifilaxis como de las lesiones cutáneas asociadas 3,39,57,60.

En este contexto, los cuidados de enfermería, están orientados principalmente a la curación de las lesiones cutáneas y a evitar que estas se infecten ${ }^{36,42,43,63}$, pues como recoge la literatura, estas lesiones son propensas a infectarse, siendo la principal causa de muerte en estos pacientes, la sepsis de origen cutáneo $28,31,33,54,57,60$. Sin embargo, no existe un protocolo estándar o pautas de actuación comunes a seguir para el cuidado de las mismas, siendo múltiples las medidas aplicadas para tal fin. 
Es fundamental la participación de enfermería en la prevención de la calcifilaxis y el reconocimiento precoz, para que las medidas terapéuticas se puedan iniciar lo antes posible, con el fin de mejorar los síntomas, conseguir la curación de las heridas y prevenir infecciones y sepsis. Enfermería también desempeña un papel importante en la educación y apoyo a paciente y familiares. El abordaje multidisciplinar para la resolución de la calcifilaxis es esencial ${ }^{46,47,49,52}$.

Como principales conclusiones de esta revisión podemos destacar:

- La calcifilaxis es una entidad que afecta principalmente a pacientes con enfermedad renal crónica en estadios avanzados y en terapia de reemplazo renal, y aunque tiene una incidencia y prevalencia muy bajas, presenta una tasa de mortalidad elevada.

- La etiopatogénesis se desconoce, aunque se han propuesto posibles mecanismos implicados, basados principalmente en las alteraciones del metabolismo, así como se han determinado varios factores de riesgo que pueden determinar su aparición.

- Se han descrito varias modalidades terapéuticas, aunque ninguna de ellas consistente.

- La prevención y detección precoz son fundamentales para evitar el desarrollo de la calcifilaxis, una entidad con mal pronóstico, debido a la mortalidad asociada a sepsis secundaria a la infección de las lesiones.

- Enfermería tiene un papel fundamental en el reconocimiento precoz, curación de las heridas, cuidado del paciente y educación sanitaria a paciente y familiares.

Recibido: 8 agosto 2015

Revisado: 16 agosto 2015

Modificado: 2 septiembre 2015

Aceptado: 12 septiembre 2015

\section{Bibliografía}

1. Verdalles-Guzmán $U$, De la Cueva $P$, Verde $E$, García-Vinuesa S, Goicoechea M, Mosse A y cols. Calcifilaxis: complicación grave del síndrome cardio-metabólico en pacientes con enfermedad renal crónica terminal (ERCT). Rev Nefrol 2008;28(1):32-36.

2. Gómez de la Fuente $E$, Vicente FJ, Álvarez JG, Naz E, Palencia SI, Pinedo F y cols. Calcifilaxis en pacientes dializados. Rev Actas Dermosifiliogr 2004;95(3):178-182.

3. Rodríguez-Villarreal I, Callejas $R$, Sánchez $M$, Laso N, Gallar P, Ortega 0 y cols. Calcifilaxis 0 arteriolopatía urémica calcificante: combinación de tratamientos. Rev Nefrol 2010;3(3):39-44.

4. Cordova KB, Oberg TJ, Malik M, Robinson-Bostom L. Dermatologic conditions seen in end-stage renal disease. Rev Semin Dial 2009;22(1):45-55.

5. Prados-Soler $M^{\mathrm{a}} \mathrm{C}$, Del Pino y Pino MD, GarófanoLópez R, Moriana-Domínguez C. Calcifilaxis severa en paciente en hemodiálisis. Rev Dial Traspl 2010;31(3):76-78.

6. Galperin TA, Cronin AJ, Leslie KS. Cutaneous manifestations of ESRD. Rev Clin $\mathrm{J} A m$ Soc Nephrol 2014;9(1):201-218.

7. García-Villasur $P$, Arranz-Martínez E, RuizGarcía A, Angulo-Ardoy M. Arteriolopatía urémica calcificante o calcifilaxia. Rev Semergen 2007;33(1):31-33.

8. Karwowski W, Naumnik B, Szczepanski M, Mysliwiec M. The mechanism of vascular calcification- a systematic review. Rev Med Sci Monit 2012;18(1):RA1-11.

9. Brewster UC. Dermatological disease in patients with CKD. Rev Am J Kidney Dis 2008;51(2):331344.

10. Virmani R, Joner M, Sakakura K. Recent highlights of ATVB. Calcification. Rev Arterioscler Thromb Vasc Biol 2014;34(7):1329-1332.

11. Sowers KM, Hayden MR. Calcific uremic arteriolopathy. Pathophysiology, reactive oxygen species and therapeutic approaches. Rev 0xid Med Cell Longev 2010;3(2):109-121. 
12. Zapata-González F, Del Río-Cobaleda DY, Ruiz AC. Calcifilaxis, reporte de un caso. Rev CES Med 2013;27(2):235-241.

13. Brandenburg VM, Kramann $R$, Specht $P$, Ketteler M. Calciphylaxis in CKD and beyond. Rev Nephrol Dial Transplant 2012;27(4):1314-1318.

14. Hayden MR, Tyagi SC, Kolb L, Sowers JR, Khanna R. Vascular ossification-calcification in metabolic síndrome, type 2 diabetes mellitus, chronic kidney disease, and calciphylaxis-calcific uremic arteriolopathy: the emerging role of sodium thiosulfate. Rev Cardiovasc Diabetol 2005;4:4.

15. Fernández E. La arteriolopatía calcificante urémica (calcifilaxis) en el contexto del síndrome metabólico y la diabetes mellitus. Perspectiva actual. Rev Nefrol 2008;28(1):20-24.

16. Mathur RV, Shortland JR, El-Nahas AM. Calciphylaxis. Rev Postgrad Med J 2001;77(911):557-561.

17. Llach F. The evolving pattern of calciphylaxis: therapeutic considerations. Rev Nephrol Dial Transplant 2001;16(3):448-451.

18. Panuncialman J, Falanga V. Unusual causes of cutaneous ulceration. Rev Surg Clin North Am 2010;90(6):1161-1180.

19. Pastor-Nieto MA. Calcifilaxia. Rev Esp Enferm Metab Óseas 2001;10(5):159-167.

20. Brewster UC. Dermatologic manifestations of end-stage renal disease. Rev Hospital Physician 2006;42(5):31-35.

21. Ross EA. Evolution of treatment strategies for calciphylaxis. Rev Am J Nephrol 2011;34(5):4607.

22. Ong S, Coulson IH. Diagnosis and treatment of calciphylaxis. Rev Skinmed 2012;10(3):166-170.

23. Rogers NM, Teubner DJ, Coates PT. Calcific Uremic Arteriolopathy: Advances in pathogenesis and treatment. Rev Semin Dial 2007;20(2):150157.

24. Polaina-Rusillo M, Sánchez-Martos MD, BiechyBaldan M M, Liebana-Cañada A. Calcifilaxis. Rev Semin Fund Esp Reumatol 2009;10(4):124-127.
25. Bhambri A, Del Rosso JQ. Calciphylaxis. A review. Rev J Clin Aesthet Dermatol 2008;1(2):38-41.

26. Esteve V, Almirall J, Luelmo J, Sáez A, Andreu X, García M. Arteriolopatía urémica calcificante (calcifilaxis): incidencia, formas de presentación y evolución. Rev Nefrol 2007;27(5):599-604.

27. Picazo M, Bover J, De la Fuente J, Sans R, Cuxart M, Matas, M. Larvas estériles como coadyuvantes al tratamiento local en una paciente con calcifilaxis proximal. Rev Nefrol 2005;25(5):559-562.

28. Capote-Moreno A, Larrañaga-Barrera E. Paratiroidectomía en pacientes con fenómenos de calcifilaxis. Rev Endocrinol Nutr 2002;49(9):308311.

29. Munar MA, Alarcón A, Bernabéu R, Morey A, Gascó J, Losada $P$ y cols. A propósito de un caso de lesiones cutáneas y calcifilaxis. Rev Nefrol 2001;21(5):501-504.

30. Marrón B, Coronel F, López-Bran E, Barrientos A. Calcifilaxia: una patogenia incierta y un tratamiento controvertido. Rev Nefrol 2001;21(6):596-600.

31. Pérez JE, Vargas JG, Echeverri JE, Rodríguez PV, Pulido JA. Calcifilaxis y enfermedad renal crónica. Rev Acta Med Colomb 2011;36(3):149-152.

32. Herrera L, Avellaneda C, Tique N. Calcifilaxis como complicación cutánea en pacientes con insuficiencia renal crónica en diálisis. Rev Med 2013;21(1):86-90.

33. Batún-Garrido JAJ, Hernández-Núñez E, MuñozPérez H. Calcifilaxis en la práctica clínica: informe de un caso con insuficiencia renal crónica terminal. Rev Med Int Méx 2014;30:721-726.

34. Morales-Jiménez EM, Rojas-Ramírez A, RocaSocarrás A. Calcifilaxis. Reporte de un caso. Rev Finlay 2013;3(4):259-263.

35. Rojas-Estrada JJ, Castelo-Villalón X, Sat FA, Semanat-Vaillant D. Arteriolopatía calcificante urémica. Rev Cub Med 2011;50(2):209-215.

36. Piña-Simón $D$, González-Horna $A$, GálvezSerrano $M^{a}$ C, Marco-García B, Rubio-González E. Actuación de enfermería ante un paciente con calcifilaxis. Rev Soc Esp Enferm Nefrol 2010;13(3):205-208. 
37. Oikawa S, Osajima A, Tamura M, Murata K, Yasuda $\mathrm{H}$, Anai $\mathrm{H}$ et al. Development of proximal calciphylaxis with penile involvement after parathyroidectomy in a patient on hemodialysis. Rev Intern Med 2004;43(1):63-68.

38. Maroulis JC, Fourtounas C, Vlachojannis JG, Siasos N, Karavias D, Chartoumpekis D et al. Calciphylaxis: a complication of end-stage renal disease improved by parathyroidectomy. Rev Hormones 2006;5(3):210-213.

39. Kakagia D, Kriki P, Thodis E, Roumeliotis A, Vargemezis V. Calcific Uremic Arteriolopathy treated with cinacalcet, paricalcitol, and autologous growth factors. Rev J Cutan Med Surg 2011;15(2):121-124.

40. Jacobs-Kosmin D, DeHoratius RJ. Calciphylaxis: An important imitator of cutaneous vasculitis. Rev Arthritis Rheum 2007;57(3):533-537.

41. Roncada EV, Abreu MA, Pereira MF, Oliveira CC, Nai GA, Soares DF. Calciphylaxis, a diagnostic and therapeutic challenge: report of a successful case. Rev An Bras Dermatol 2012;87(5):752-755.

42. Marques SA, Kakuda AC, Mendaçolli TJ, Abbade LP, Marques ME. Calciphylaxis: a rare but potentially fatal event of chronic kidney disease. Case report. Rev An Bras Dermatol 2013;88(6 Suppl 1):44-47.

43. Hayashi M. Calciphylaxis: diagnosis and clinical features. Rev Clin Exp Nephrol 2013;17(4):498503.

44. Mohammed IA, Sekar V, Bubtana AJ, Mitra S, Hutchison AJ. Proximal calciphylaxis treated with calcimimetic "cinacalcet". Rev Nephrol Dial Transplant 2008;23(1):387-389.

45. Velasco N, MacGregor MS, Innes A, Mackay IG. Successful treatment of calciphylaxis with cinacalcet- an alternative to parathyroidectomy? Rev Nephrol Dial Transplant 2006; 21(7):19992004.

46. Bliss DE. Calciphylaxis: What nurses need to know. Rev Nephrol Nurs J 2002;29(5):433-444.

47. Headley CM, Wall B. ESRD- associated cutaneous manifestations in a hemodialysis population. Rev Nephrol Nurs J 2002;29(6):525-539.
48. Garini G, Galletti E, Ricci R, Vaglio A, Buzio C. Calciphylaxis: evolving concepts. Rev Intern Emerg Med 2006;1(4):320-322.

49. Carter T, Ratnam S. Calciphylaxis: A devastating complication of derangements of calciumphosphorus metabolism- $A$ case report and review of the literatura. Rev Nephrol Nurs J 2013;40(5):431-435.

50. Sprague SM. Painful Skin Ulcers in a Hemodialysis Patient. Rev Clin J Am Soc Nephrol 2014;9(1):166-173.

51. Hayashi M, Takamatsu I, Kanno Y, Yoshida T, Abe T, Sato Y. A case-control study of calciphylaxis in Japanese end-stage renal disease patients. Rev Nephrol Dial Transplant 2012;27(4):1580-1584.

52. González-Horna A, Piña-Simón D, GálvezSerrano M, Gago-Gómez $M^{a}$ C, Sánchez-Mangas JR, Martínez-Gómez S y cols. Manifestaciones dérmicas en hemodiálisis. Cuidados de enfermería ¿nuevo enfoque? Rev Soc Esp Enferm Nefrol 2005;8(4):323-326.

53. Torregrosa JV, Durán $C E$, Barros $X$, Blasco $M$, Arias M, Cases A y cols. Tratamiento eficaz de la arteriolopatía urémica calcificante con bifosfonatos. Rev Nefrol 2012;32(3):329-334.

54. Mazhar AR, Johnson RJ, Gillen D, Stivelman JC, Ryan MJ, Davis CL et al. Risk factors and mortality associated with calciphylaxis in end-stage renal disease. Rev Kidney Int 2001;60(1):324-332.

55. Farah M, Crawford RI, Levin A, Chan-Yan C. Calciphylaxis in the current era: emerging "ironic" features? Rev Nephrol Dial Transplant 2011;26(1):191-195.

56. Fine $A$, Fontaine B. Calciphylaxis: the beginning of the end? Rev Perit Dial Int 2008;28(3):268-270.

57. Hammawa-Malabu U, Manickam V, Kan G, Lynette-Doherty S, Singh-Sangla K. Calcific uremic arteriolopathy on multimodal combination therapy: still unmet goal. Rev Int $\mathrm{J}$ Nephrol 2012;2012: 1-6.

58. New N, Mohandas J, John GT, Ratanjee S, Healy $H$, Francis $L$ et al. Calcific uremic arteriolopathy in peritoneal dialysis population. Rev Int J Nephrol 2011;2011: 1-9. 
59. Nigwekar SU, Brunelli SM, Meade D, Wang W, Hymes J, Lacson EJ. Sodium thiosulfate therapy for calcific uremic arteriolopathy. Rev Clin J Am Soc Nephrol 2013; 8(7):1162-1170.

60. Zitt $E$, Konig $M$, Vychytil $A$, Auinger $M$, Wallner $M$, Lingenhel $G$ et al. Use of sodium thiosulfate in a multi-interventional setting for the treatment of calciphylaxis in dialysis patients. Rev Nephrol Dial Transplant 2013;28(5):1232-1240.

61. Podymow T, Wherrett C, Burns KD. Hyperbaric oxygen in the treatment of calciphylaxis. Rev Nephrol Dial Transplant 2001;16(11):2176-2180.

62. Llach F. The evolving clinical features of calciphylaxis. Rev Kidney Int 2003;63 Supl 85:S122-S124.

63. Lerma-García D, Lerma-García B, EcheverríaEciolaza MI, Sureda-Parera J. Cuidados integrales de enfermería a pacientes con úlceras por calciphylaxis en tratamiento con hemodiálisis: A propósito de un caso. En: Comunicaciones Presentadas al XXXIV Congreso Nacional SEDEN. Ibiza;2009.p.21-26.
64. Torregrosa JV, Bover J, Cannata-Andía J, Lorenzo V, De Francisco ALM, Martínez I y cols. Recomendaciones de la Sociedad Española de Nefrología para el manejo de las alteraciones del metabolismo óseo-mineral en los pacientes con enfermedad renal crónica (S.E.N.-MM). Rev Nefrol 2011;31(Suppl 1):3-32.

65. National Kidney Foundation. K/DOQI Clinical Practice Guidelines for Bone Metabolism and Disease in Chronic Kidney Disease. Rev Am J Kidney Dis 2003;42(4 Suppl 3):S1-S201.

66. National Kidney Foundation. Clinical Practice Guidelines for Nutrition in Chronic Renal Failure. Rev Am J Kidney Dis 2000;35(6 Suppl 2):S1S140. 